\title{
Learning When to Trust Which Experts
}

\author{
David Helmbold ${ }^{1}$ and Stephen Kwek ${ }^{2}$ and Leonard Pitt ${ }^{3}$ \\ 1 University of California at Santa Cruz, Department of Computer Science, Santa \\ Cruz, CA, 95064, U.S.A., Email: dph@cse.ucsc.edu \\ 2 Department of Computer Science, Washington University, St. Louis, MO 63130, \\ Email: kwek@cs.wustl.edu \\ 3 University of Illinois at Urbana-Champaign, Department of Computer Science, \\ Urbana, IL 61801, U.S.A., Email: pitt@cs.uiuc.edu
}

\begin{abstract}
The standard model for prediction using a pool of experts has an underlying assumption that one of the experts performs well. In this paper, we show that this assumption does not take advantage of situations where both the outcome and the experts' predictions are based on some input which the learner gets to observe too. In particular, we exhibit a situation where each individual expert performs badly but collectively they perform well, and show that the traditional weighted majority techniques perform poorly.

To capture this notion of 'the whole is often greater than the sum of its parts', we propose an approach to measure the overall competency of a pool of experts with respect to a competency class or structure. A competency class or structure is a set of decompositions of the instance space where each expert is associated with a 'competency region' in which we assume he is competent. Our goal is to perform close to the performance of a predictor who knows the best decomposition in the competency class or structure where each expert performs reasonably well in its competency region. We present both positive and negative results in our model.
\end{abstract}

\section{Introduction}

\subsection{The Deficiency of the Standard Weighted Majority Techniques for Prediction Using a Pool of Experts}

In [LW94], Littlestone and Warmuth study the problem of making on-line prediction using a pool of experts. In their model, the learner faces a (possibly infinite) sequence of trials, with a boolean prediction to be made in each trial, and the goal of the learner is to make few mistakes. The learner is allowed to make his prediction by observing how a given pool of experts predict. The underlying assumption is that at least one of these experts will perform well but the learner does not know which one.

They propose the weighted majority algorithm which works as follows. A weight is associated with each expert. Initially, all the weights are set to one. The learner predicts 0 if the sum of the weights of all the experts that predict 0 is greater than that of the experts that predict 1, otherwise the learner predicts 
1. When a mistake is made, the learner simply multiplies the weights of those experts that predict wrongly by some fixed non-negative constant $\beta$ smaller than one. The following mistake bounds are obtained for the weighted majority algorithm.

Theorem 1. [LW94] Given a pool of experts $\mathcal{E}$, on any (possibly infinite) sequence of trials, the weighted majority algorithm makes at most

a) $O(\log |\mathcal{E}|+\eta)$ mistakes, if one of the experts makes at most $\eta$ mistakes.

b) $O(\log (|\mathcal{E}| / k)+m / k)$ mistakes, if there is a subset of $k$ experts making a total of $m$ mistakes.

Observe that in this weighted majority algorithm, the weight of an expert after making $l$ mistakes is $\beta^{l}$ and hence, it is also known as the weighted majority algorithm using an exponcntial weighting scheme. Recently, an alternate weighting scheme, called the binomial weighting scheme, has been suggested by Cesa-Bianchi et al. [CBFHW95] and is shown to have a better mistake bound.

Suppose the experts' predictions and the actual outcome depend on some input (instance) in each trial. Notice that neither the binomial weighting scheme nor the exponential weighting scheme makes use of the instance when calculating the weights of the experts. This can be a serious disadvantage when the learnor can use the instances to determine which experts are likely to predict correctly.

To illustrate this, consider the following example with a boolean instance space $\{0,1\}^{n}$ and two experts, $E_{0}$ and $E_{1}$ that always give opposite predictions. If $E_{0}$ makes at most $m_{0}$ mistakes if one crucial component of the instance is set to 0 , and $E_{1}$ makes at most $m_{1}$ mistakes when that component is set to 1 , then the weighted majority algorithm can be forced to make a mistake on almost every point in the instance space (more precisely, $2^{n}-\left|m_{0}-m_{1}\right|$ mistakes), even if it uses table lookup to remember all of its previous mistakes. However, if the learner uses $E_{0}$ 's predictions when the crucial input component was set to 0 and $E_{1}$ 's predictions otherwise, then the learner makes at most $m_{0}+m_{1}$ mistakes when it maintains a table of counterexamples. In other words, although neither expert is very competent, collectively they become competent if we consider restricting the use of each expert $E_{i}$ to the appropriate subset of the instance space.

Unfortunately, the algorithms that employ the weighted majority techniques do not take advantage of this situation. Hence, we propose two approaches that capture this notion of "the whole is often greater than the sum of its parts.":

Competency Class: In the first approach, each expert's area of expertise is assumed to be representable as a function chosen from some known "competency class" (of concepts).

Competency Structure: Alternatively, the instance space is partitioned into regions and each expert is associated with one such region as his area of expertise. Often it is assumed that the partition is representable as a $k$ valued function taken from some known class of functions, which we call competency structure. 
With this view, the learning problem may be seen as that of determining the area of expertise of each expert. However, we illustrate later that this view is not necessarily the best.

\subsection{The Competency Model}

Suppose we have a collection of $k$ experts $\mathcal{E}=\left\{E_{1}, \cdots, E_{k}\right\}$ where each expert can be viewed as a boolean function on some finite instance space $\mathcal{X}$. Let $f$ be an arbitrary target concept (boolean function) defined over $\mathcal{X}$. The learner's goal is to use the set of experts in order to approximate the target $f$. We make no assumption on the set of experts $\mathcal{E}$ or the target $f$ other than they are functions of the instance space. As in other settings focusing on prediction with expert advice, we measure the performance of the learner relative to the "goodness" of the set of experts.

A covering $C=\left\langle c_{1}, \cdots, c_{k}\right\rangle$ is an ordered set of $k$ subsets of the instance space with the property that the union of the $k$ subsets is the entire instance space $\mathcal{X}$. The covering assigns the competency region $c_{i} \subseteq \mathcal{X}$ to each expert $E_{i}$ such that ideally, each expert should be competent (have low incompetence) in its competency region. This motivates the following definition. The incompetence of an expert $E_{i}$ with respect to the covering $C=\left\langle c_{1}, \cdots, c_{k}\right\rangle$ and target $f$, denoted by incom $\left(E_{i}, C, f\right)$, is defined as the number of mistakes $E_{i}$ makes over its competency region $c_{i}$. That is,

$$
\operatorname{incom}\left(E_{i}, C, f\right)=\left|\left\{x \in c_{i}: E_{i}(x) \neq f(x)\right\}\right|
$$

The incompetence of the entire collection of experts $\mathcal{E}$ with respect to the covering $C$ and target function $f$, written as $\operatorname{incom}(\mathcal{E}, C, f)$, is defined as

$$
\operatorname{incom}(\mathcal{E}, C, f)=\sum_{i=1}^{k} i n \operatorname{com}\left(E_{i}, C, f\right) \text {. }
$$

Intuitively, if the learner makes his prediction on each input $x$ by taking a majority vote on the predictions of the set of experts whose competency regions in the covering $C$ contain $x$, then the maximum number of mistakes that the learner makes is incom $(\mathcal{E}, C, f)$ assuming that at most one mistake is made on each instance. (This can be done by storing the counterexamples in a table, provided the incompetence of each expert $E_{i}$ w.r.t. $C$ is not too large.) The following property follows immediately from the definition of incompetence.

Property 2. If $C^{\prime}=\left\langle c_{1}, \cdots \cdot c_{k}\right\rangle$ and $C^{\prime}=\left\langle c_{1}^{\prime}, \cdots, c_{k}^{\prime}\right\rangle$ are two coverings of the instance space where $c_{i} \subseteq c_{i}^{\prime}$ for each $1 \leq i \leq k$ then $\operatorname{incom}(\mathcal{E}, C, f) \leq$ $\operatorname{incom}\left(\mathcal{E}, C^{\prime}, f\right)$.

Note that the competency regions in a covering can overlap and that the incompetence measure tends to penalize coverings with large overlaps, since the mistakes of the experts on the overlapping region are added together. 
Suppose no assumptions are made about the competency regions. Consider two 'experts' (or more appropriately idiots) $E_{0}$ and $E_{1}$ where $E_{0}$ always predict 0 regardless of what the instance is, and $E_{1}$ always predict 1 . Suppose we do not place any restriction on the competency regions allowed. For any boolean concept $f$, if we let $c_{i}$ be the part of the instance space that is classified as $i$ by $f$ then $C=\left\langle c_{0}, c_{1}\right\rangle$ forms an optimum covering where incom $\left(\left\{E_{0}, E_{1}\right\}, C, f\right)=0$ ! In other words, these two experts form a competent team in predicting any boolean concept, which means any positive result in our prediction model would imply that we can learn any boolean concept. Thus, learning arbitrary coverings is as hard as learning arbitrary functions. Therefore, we must make assumptions about the competency regions, even if the prediction strategies of the experts are simple. We use two alternative ways of restricting the possible coverings to make the learning of competency regions tractable:

Competency Class: We first consider restrictions on the competency regions used. Let $\mathcal{R}$ be a class of concepts. A covering $C=\left\langle c_{1}, \cdots, \cdot c_{k}\right\rangle$ is in the competency class induced by $\mathcal{R}$, written $\mathcal{C}_{\mathcal{R}}$, if and only if each $c_{i}$ is a member of $\mathcal{R}$.

Competency Structure: When the sets of a covering are disjoint, the covering essentially maps each instance to an expert. Thus, the covering may be viewed as a classification function $C(x)=i$ where expert $E_{i}$ is good on instance $x$. Our second kind of restriction allows only those coverings which correspond to certain mappings. Given a mapping class $\mathcal{M}$, the competency structure $\mathcal{S}_{\mathcal{M}}$ induced by $\mathcal{M}$ is the set of all coverings consistent with a mapping in $\mathcal{M}$.

For example, suppose DL is the class of decision lists. Then $\mathcal{C}_{\mathrm{DL}}$ is the class of coverings where the competency regions in each admissible covering can be expressed using binary decision lists and $\mathcal{S}_{\mathrm{DL}}$ is the class of coverings which partition the instance space using a $k$-valued decision list.

The joint incompetence of $\mathcal{E}$ with respect to a competency class or structure $\mathcal{C}$, and target $f$, incom $(\mathcal{E}, \mathcal{C}, f)$, is the minimum incompetence of the entire collection of experts $\mathcal{E}$ over all coverings of $\mathfrak{X}$ in $\mathcal{C}$. That is,

$$
\operatorname{incom}(\mathcal{E}, \mathcal{C}, f)=\min _{C \in \mathcal{C}} \operatorname{incom}(\mathcal{E}, C, f) \text {. }
$$

We say that $\mathcal{E}$ is completely competent if $\operatorname{incom}(\mathcal{E}, \mathcal{C}, f)=0$.

An optimum covering in some competency class or structure $\mathcal{C}$ is any covering $C^{\text {opt }}=\left\langle C_{1}^{\text {opt }}, \cdots, C_{k}^{\text {opt }}\right\rangle$ such that $\operatorname{incom}\left(\mathcal{E}, C^{\text {opt }}, f\right)=\operatorname{incom}(\mathcal{E}, \mathcal{C}, f)$. In other words, if the learner predicts using an optimal covering of $\mathcal{X}$ then its worst case number of mistakes is at most $\operatorname{incom}(\mathcal{E}, \mathcal{C}, f)$. For instance, in the example of Section 1.1, the joint incompetence of the two experts with respect to the competency class of single literals ${ }^{4}$ is at most $m_{1}+m_{2}$ where the competency regions are defined by the crucial component of the input. From here on, we shall

\footnotetext{
${ }^{4}$ Actually, for this simple example we could also use the competency class of disjunctions or the competency structure of decision lists.
} 
simply refer to the joint incompetence of $\mathcal{E}$ with respect to $\mathcal{C}$ as the incompetence of $\mathcal{E}$.

In our new model, which we call the competency model, the learner is given a pool of experts $\mathcal{E}=\left\{E_{1}, \cdots, E_{k}\right\}$ and we assume there exists a good covering from some known competency class or structure $\mathcal{C}$. The learning process consists of a sequence of trials as in Littlestone and Warmuth's model. On the th trial, an instance $x^{t} \in \mathcal{X}$ is given to the learner, as well as the experts' predictions for $x^{t}$. The learner then predicts how the target concept $f$ classifies $x^{t}$ based on the experts' predictions and its past experience. After the prediction is made, the learner is told $f\left(x^{t}\right)$, the correct classification of $x^{t}$. If the prediction is incorrect, then the learner has made a mistake. This feedback can then be used to improve the learner's future predictions. The learner is said to be able to learn the competency class or structure $\mathcal{C}$ over an instance space $\mathcal{X}$ if the following criterion is met.

Learning criterion in the competency model: For any target concept $f$ and any set $\mathcal{E}$ of experts, if the number of mistakes the learner makes on any sequence of trials is bounded by some polynomial poly $(|\mathcal{E}|$, incom $(\mathcal{E}, \mathcal{C}, f)$, complex $\left(\mathfrak{X}^{\prime}\right)$, complex $\left(C^{\text {opt }}\right)$ ) for some competency class or structure $\mathcal{C}$ then we say $\mathcal{C}$ is learnable. Here, complex $(\mathcal{X})$ and complex $\left(C^{\text {opt }}\right)$ are some appropriate complexity measures of the instance space $\mathcal{X}$ and an optimal covering $C^{\text {opt }}$ from $\mathcal{C}$. For example, if $\mathcal{C}$ is the class of disjunctions of boolean literals then complex $(\mathcal{X})$ is the number of variables and complex $\left(C^{\text {opt }}\right)$ is the total number of literals that appear in $C^{o p t}$. We say the learner is efficient if the time it consumes in each trial is bounded by some polynomial in $|\mathcal{E}|$. $\operatorname{incom}(\mathcal{E}, \mathcal{C}, f)$, complex $(\mathcal{X})$ and complex $\left(C^{\text {opt }}\right)$.

Furthermore, we say $\mathcal{C}$ or $C^{\text {opt }}$ is identifiable if we can identify each expert's competency region in the optimum covering using $\mathcal{C}$ without making more than some polynomial poly $y_{i}\left(|\mathcal{E}|\right.$, incom $(\mathcal{E}, \mathcal{C}, f)$, complex $\left(\mathcal{X}^{\prime}\right)$, complex $\left.\left(C^{\text {opt }}\right)\right)$ number of mistakes.

It is clear that identifiability implies learnability. However, it is not clear, and probably not true, that learnability implies identifiability. There are situations where identifiability is desirable. For example, you may not have the resources to employ all the experts but are able to observe their past performance. In this case, you would like to infer their competency regions and select the subset of experts that suits your need.

Notice that the standard model used in the weighted majority algorithm is a specialization of the competency model where the competency class $\mathcal{C}$ consists of exactly two elements, the entire instance space $\mathcal{X}$ and the empty set. However, we have also relaxed the criterion for learning by not insisting that the mistake bound be polynomial in $\log k$ (as is the weighted majority's bound) but in $k$ instead. This is reasonable if the number of experts, $k$, is not extremely large. 


\subsection{Organization of This Paper}

In Section 2, we present an efficient algorithm for learning the competency class (structure) of intervals in a finite linearly ordered instance space. Note that there is no distinction between competency class and structure when the competency regions are intervals, as they can be easily shown to be equivalent. The algorithm can be easily generalized to learn fixed depth $d$-ary decision trees (omitted here).

In Section 3, we begin by presenting an efficient algorithm for identifying the competency class of disjunctions of boolean literals. We do not require the instance space to be $\{0,1\}^{n}$ but may be a subset of $\{0,1\}^{n}$, as long as our optimum covering contains the subset of $\{0,1\}^{n}$ that may occur in our trials (a.k.a. effective instance space). We then continue to exhibit a simple learning algorithm for learning the same competency class with a smaller mistake bound. In doing so, we illustrate that identifying an optimum covering may not be the best way of learning a competency class. Both algorithms are based on Littlestone's [Lit88] WINNow algorithm for learning $r$-literal disjunctions which has the ability to pick up the relevant attributes quickly even when there are (exponentially) many irrelevant attributes.

Our results also imply that the competency class of $k$-DNF formulas is identifiable and learnable when $k$ is fixed. At first glance, it may seem that our results also imply that the concept class of DNF formulas is learnable in the on-line mistake bound model by having an optimum covering consisting of one expert, which always predicts true, for each term $t$ in the target DNF formula with compctency region $t$. Unfortunately, our definition of covering requires that the entire effective instance space be covered. One could cover the false instances by adding an extra expert who always predicts false and whose competency region is the entire domain. Unfortunately, this may increase the incompetence of the experts dramatically.

Knowing that we can identify the competency class of disjunctions by modifying Littlestone's WINNow algorithm, one may ask whether we can identify the competency class of linear threshold functions over the boolean instance space, since they can be learned in the standard on-line model using Winnow as well. Unfortunately, it is not clear how we can adapt Winnow to do so. In fact, we show in Section 4 that identifying the competency class of conjunctions, which is a subclass of linear threshold functions, is as hard as learning decision trees in the on-line mistake bound model with a constant number of lies. The latter result, though not known to be impossible, is a difficult open problem.

In Section 5, we modify a previously known ${ }^{5}$ on-line algorithm for learning decision lists to obtain an efficient learning algorithm for the decision list competency structures. We conclude in Section 6 with some open problems in this new frontier of prediction using experts' advice.

Throughout this paper, we assume implicitly that all the counterexamples seen are stored in a table along with their classifications. If an instance happens to be in the table, then the learner predicts with the correct classification that is stored in the table.

\footnotetext{
${ }^{5}$ This technique is due to Littlestone (private communication).
} 


\section{Learning the Interval Competency Class or Structure}

In this section, we assume that the (finite) instance space is linearly ordered and consider the competency class $\mathcal{C}_{\mathcal{I}}$ induced by the class of intervals $\mathcal{I}$. Since the domain is linearly ordered, we can assume that the instance space is numbered $1, \ldots d$. The class of intervals, $\mathcal{I}$, contains all subsets of the instance space of the form $i, \ldots, j$. A covering is in $\mathcal{C}_{\mathcal{I}}$ if and only if each $c_{i}$ is in $\mathcal{I}$.

Note that this competency class includes coverings with overlapping intervals. However, it is easy to see that there will always be an optimum covering with disjoint intervals. This follows from Property 2 and the fact that for any pair of intervals $I$ and $I^{\prime}$, there are two disjoint intervals $\widehat{I} \subseteq I$ and $\widehat{I}^{\prime} \subseteq I^{\prime}$ such that $I \cup I^{\prime}=\widehat{I} \cup \widehat{I}^{\prime}$. Therefore learning the competency class of intervals is closely related to learning the competency structure which maps a (possibly empty) interval of the domain to each expert.

We now restrict our attention to coverings with disjoint intervals. When there are $k$ experts and the instance space has $d$ points then there are $\left(\begin{array}{l}d+1 \\ k-1\end{array}\right) k ! \leq(d k)^{k}$ such coverings. If the set of experts is completely competent, then the halving algorithm [LW94] can be used to learn the competency class while making at most $k \log d k$ mistakes. We refine this approach to overcome two difficulties the halving algorithm is not generally efficient, and the experts may not be completely competent.

Assume that the $\operatorname{incom}\left(\mathcal{E}, \mathcal{C}_{\mathcal{I}}, f\right)=\eta$, so that the optimal covering mislabels $\eta$ points. We extend the set of coverings to include one which is perfect and use dynamic programming to efficiently implement the conservative halving algorithm on this extended class of coverings. First we extend the covering by closing it to $\eta$ exceptions (at the end of the section we describe a standard doubling-trick for when $\eta$ is unknown). This ensures that one of the possible coverings is perfect. To speed up our algorithm, we also allow several of the $k$ intervals in the covering to be associated with the same expert. Thus we are actually learning a more general concept structure which can be represented as a $k$-decision list with decisions of the form "is $x \leq i$ ?"

We implement the conservative halving algorithm by counting the number of hypotheses that correctly label those previously seen points in the algorithm's table and also label the new point 0 , and compare this with the number of hypotheses that correctly label the points in the table while labeling the new point 1 . The halving algorithm predicts with the label for the new point that has the larger count, or arbitrarily if the counts are equal. Thus the halving algorithm can be implemented efficiently whenever one can efficiently count the number of hypotheses that correctly label a given sample. We show how dynamic programming can be used to do this counting.

Since there are $\left(\begin{array}{l}d \\ \eta\end{array}\right)$ ways of selecting the $\eta$ exceptions, $\left(\begin{array}{l}d+1 \\ k-1\end{array}\right)$ ways of partitioning the instance space into $k$ subintervales and $k^{k}$ ways of assigning each subinterval to an expert, there are $\left(\begin{array}{l}d \\ \eta\end{array}\right)\left(\begin{array}{l}d+1 \\ k-1\end{array}\right) k^{k} \leq d^{\eta}(d k)^{k}$ hypotheses in the extended class. Let $p_{1}, p_{2}, \ldots, p_{r}$ be a set $r$ labeled instances in ascending order. Define the function \# $(c, i, j, n)$ (see Figure 1$)$ to be the number of ways that $1, \ldots, p_{c}$ can be covered with $i$ intervals such that the last interval uses expert 
$E_{j}$ and the covering makes $n(\leq \eta)$ mistakes on points $1, \ldots, p_{c}$. The following lemma gives a recurrence relation on the function $\#(c, i, j, n)$ which allows us to compute \# $(c, i, j, n)$ for all feasible choices of $c, i, j$ and $n$, using dynamic programming ${ }^{6}$ in $O\left(r k^{2} \eta\right)$ time.

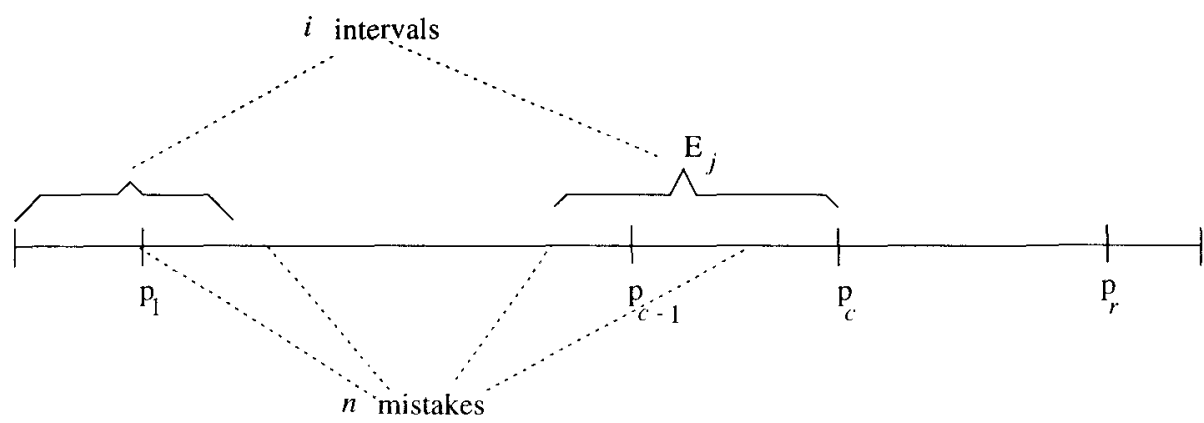

Fig. 1. The function \#(c,i,j,n).

Lemma 3. The function \# $(c, i, j, n)$ satisfies the following recurrences: If expert $j$ agrees with the label of $p_{r}$ then

$$
\begin{aligned}
& \#(c, i, j, n)=\#(c-1, i, j, n)+ \\
& \quad \sum_{s=1}^{\imath-1}\left(\begin{array}{c}
p_{c}-p_{c-1} \\
s
\end{array}\right) k^{s-1} \sum_{n^{\prime}=0}^{\eta}\left(\begin{array}{c}
p_{r}-p_{c-1}-1 \\
n^{\prime}
\end{array}\right) \sum_{j^{\prime}=1}^{k} \#\left(c-1, i-s, j^{\prime}, \eta-n^{\prime}\right) .
\end{aligned}
$$

If expert $j$ disagrees with the label of $p_{e}$ there will be a mistake on point $p_{c}$, and

$$
\begin{aligned}
& \#(c, i, j, n)=\#(c-1, i, j, n-1)+ \\
& \quad \sum_{s=1}^{i-1}\left(\begin{array}{c}
p_{c}-p_{c-1} \\
s
\end{array}\right) k^{s-1} \sum_{n^{\prime}=0}^{\eta-1}\left(\begin{array}{c}
p_{c}-p_{c-1}-1 \\
n^{\prime}
\end{array}\right) \sum_{j^{\prime}=1}^{k} \#\left(c-1, i-s, j^{\prime}, \eta-1-n^{\prime}\right) .
\end{aligned}
$$

Proof: The first term \# $(c-1, i, j, n)$ on the right-hand side of Equation (1) is to count those configurations where the last interval covers both $p_{c-1}$ and $p_{c}$. The second term counts the number of configurations where the last interval covers $p_{c}$ but not $p_{c-1}$.

The number of intervals, $s$, between $p_{c-1}$ and $p_{c}$, can range from 1 to $i-1$. For each choice of $s$, there are $\left(\begin{array}{c}p_{c}-p_{c-1} \\ s\end{array}\right) k^{s-1}$ choices of $s$ intervals in the half open

${ }^{6}$ The algorithm consists of four nested loops iterating through all possible choices of $c, i, n$ and $j$ in that order. 
interval $\left(p_{c-1}, p_{c}\right]$ and assignments of experts to these intervals. (Note that the last interval has already been assigned to expert $E_{j}$.) Furthermore, the number of exceptions, $n^{\prime}$, in the open interval $\left(p_{c-1}, p_{c}\right)$ ranges from 0 to $\eta$ and for each choice of $n^{\prime}$, there are $\left(\begin{array}{c}p_{c}-p_{c-1}-1 \\ n^{\prime}\end{array}\right)$ ways of selecting $n^{\prime}$ exceptions in the open interval $\left(p_{c-1}, p_{c}\right)$. The innermost summation is simply counting the number of ways of covering the remaining subinterval in $\left[1, p_{c}\right]$ after fixing both $s$ and $n^{\prime}$, and the fact that there are $k$ possible experts that can be assigned to the next to last interval.

Equation (2) can be derived similarly.

The number of coverings that are consistent with the $r$ labeled sample is

$$
\sum_{i=1}^{k} \sum_{j=1}^{k} \sum_{n=0}^{\eta}\left[\#(r, i, j, k) \cdot \sum_{\alpha=1}^{k-i+1}\left(\begin{array}{c}
d-p_{r} \\
\alpha
\end{array}\right) k^{\alpha-1} \cdot \sum_{\beta=1}^{\eta-n}\left(\begin{array}{c}
d-p_{r} \\
\beta
\end{array}\right)\right]
$$

The three outermost summations are to count the ways of specifying the portions of the coverings that cover the closed interval $\left[1, p_{r}\right]$ using $i$ intervals, with the last interval being assigned to expert $j$, and the $n$ exceptions on the closed interval $\left[1, p_{r}\right]$. The sum $\sum_{\alpha=1}^{k-i+1}\left(\begin{array}{c}d-p_{r} \\ \alpha\end{array}\right) k^{\alpha-1}$ counts the number of ways of covering the remaining points $p_{r}+1, \cdots, d$ using at most $k-i+1$ intervals where the leftmost interval is an extension of the last interval in the covering of the interval $\left[1, p_{r}\right]$, while the sum $\sum_{\beta=1}^{\eta-n}\left(\begin{array}{c}d-p_{r} \\ \beta\end{array}\right)$ counts the number of ways of selecting up to $\eta-n$ points, from $p_{r}+1, \cdots, d$, where exceptions are made. Thus, we can count the number of coverings consistent with the sample, which allows us to implement the halving algorithm. The number of mistakes made by the halving algorithm is at most the logarithm of the number of hypotheses, or $\eta \log d+k \log (d k)$, and this quantity bounds the number of points $r$ that must be stored in the table.

The above assumed that the learner knew $\eta$, the incompetence of $\mathcal{E}$. A simple doubling trick can be used to avoid this difficulty. The learner first assumes that $\eta \leq 2$, and then when that assumption is violated, we restart and assume that $\eta=4$, and so on. The number of mistakes made is now bounded by $2 \eta \log d+k \log (d k) \log \eta$. This shows that the class of intervals is learnable in the competency model. If all the \#(r,i,j,n)'s for all possible choices of $i, j$ and $n$ have been computed using Lemma 3, then the sum in Equation (3) can be computed in $O\left(k^{3} \eta^{2}\right)$ time. Thus, we have the following theorem.

Theorem 4. Suppose a pool of $k$ experts has joint incompetence $\eta$ w.r.t. the competency class (structure) of intervals. We can efficiently learn the competency class or structure of $k$ intervals with mistake bound $2 \eta \log d+k \log (d k) \log \eta$ and running time $O\left(k^{3} \eta^{2}+r k^{2} \eta\right)$ per trial. Here, $r$ is the number of mistakes made so far.

\section{Learning and Identifying the Competency Class of Disjunctions}

In this section, we show that identifying an optimum covering may not be the best way of learning a competency class of disjunctions of literals. 
When the instance space is a subset of the boolean hypercube $\{0,1\}^{n}$, the competency class of disjunctions consists of all coverings where each expert is associated with the subset of the space satisfying a disjunction of literals. An expert can be associated with the empty set through the use of the empty disjunction. As in the previous section, we assume that the incompetence of the experts is some value $\eta$ since we can guess $\eta$ using the doubling trick described previously.

The main idea of our algorithm for learning the competency class of disjunctions of literals is to run an algorithm, which is a hybrid of Littlestone's Winnow1 and Winnow2 a]gorithms [Lit88], for each expert $E_{i}$ to learn its competency region $C_{i}^{\text {opt }}$ in an optimum covering $C^{\text {opt }}$. The learner maintains a hypothesis $h_{i}$ for the disjunction representing each $C_{i}^{\text {opt }}$ in the optimal covering. These hypotheses are linear threshold functions using one weight per variable, so that an instance $x$ is in $h_{i}$ if and only if $\sum_{j=1}^{n} w_{i j} x_{j} \leq k n$ (recall that $k$ is the number of experts). Initially, all the weights are set to 1 , as in WinNow. For each expert $E_{i}$, we also maintain a counter $\alpha_{i j}$ for each literal $x_{j}$ which counts the number of mistakes $E_{i}$ made in the previous trials when $x_{j}$ is set to 1 .

Given an input $x$ (assuming $\mathrm{jt}$ is not a counterexample which the learner has already seen), the learner first checks whether $x$ is cover d by (contained in) any of the its current hypotheses $h_{i}$. If $r$ is covered, then for $j \in\{0,1\}$, let $\mathcal{E}_{j}$ be the set of experts that predict $j$ on $x$ and whose corresponding $h_{i}$ s contain $x$. Otherwise, the learner sets $\mathcal{E}_{j}, j \in\{0,1\}$, to be the set of experts that predirt $j$. The learner than predicts 0 if $\left|\mathcal{E}_{0}\right| \geq\left|\mathcal{E}_{1}\right|$ and 1 otherwise. If a mistake is made, say we predicted $y$ which is wrong then we update the weights of our hypotheses according to the following rules:

$\eta$-elimination: Suppose $x$ is covered. For each expert $E_{i}$ in $\mathcal{E}_{y}$, if $x_{j}=1$ then increment $\alpha_{\imath j}$ by one and check if $\alpha_{i j}>\eta$. If yes, then we can conclude that $x_{3}$ cannot appear in the disjunction describing the competency region of $E_{i}$ (since $E_{i}$ can make at most $\eta$ mistakes on points in its competency region) and hence, we can eliminate $x_{j}$ from $h_{i}$ by setting $w_{i j}=0$. Otherwise, we simply do a demotion as in WINNOW 1 on $w_{i j}$ by dividing it by $a$.

Promotion: Suppose $x$ is not covered. In this case, we perform a promotion step on the weights of $h_{i}$ for each $i$ such that $E_{i} \in \mathcal{E}_{\bar{y}}$. The idea here is that at less one of the $h_{i}$ 's is improved unless all the experts whose competency regions in the optimum covering cover $x$ predict incorrectly which happens at most $\eta$ times.

We begin by proving some simple lemmas similar to those used in proving the mistake bound of WinNow [Lit88]. Let $u$ and $v$ be the number of promotion steps and $\eta$-elimination steps, respectively. Then the number of mistakes is simply the sum of $u$ and $v$.

\section{Lemma 5.}

$$
v \leq \frac{\alpha}{\alpha-1}+\alpha k u
$$


Proof: Consider the sum

$$
S=\sum_{i=1}^{k} \sum_{j=1}^{n} w_{i j}
$$

$S$ is always non-negative. Initially, $S=k n$.

Suppose we make a mistake by predicting the classification of $x$ to be $y$. An $\eta$-elimination step occurs only if $x$ is covered and hence, only if $\left|\mathcal{E}_{y}\right| \geq 1$. Therefore, before the $\eta$-elimination,

$$
\sum_{i: E_{i} \in \mathcal{E}_{y}} \sum_{j} w_{i j} x_{j} \geq\left|\mathcal{E}_{y}\right| k n \geq k n .
$$

After an $\eta$-elimination, the above sum is reduced by a factor of $1-\alpha^{-1}$. When a promotion step occurs, the sum $\sum_{j} w_{i j}$ either does not change or increases no more than $(\alpha-1) k n$. Thus,

$$
0 \leq S \leq k n+k u(\alpha-1) k n-v k n\left(1-\alpha^{-1}\right)
$$

which gives us the desired bound.

Lemma 6. For all $i, j, w_{i j} \leq \alpha k n$.

Proof: Since a weight is promoted only if it is not greater than $k n$.

Lemma 7. After $u$ promotion steps and any number of $\eta$-elimination steps, there exists a pair $i$ and $j$ such that

$$
\log _{\alpha} w_{i j} \geq \frac{u-\eta L-\eta}{L}
$$

where $L$ is the sum, over all $C_{i}^{o p t} s$, of the number of literals ${ }^{7}$ in the disjunction. describing $C_{i}^{\text {opt }}$.

Proof: Suppose, $C_{i}^{\text {opt }}=x_{j_{1}^{2}} \vee \cdots \vee x_{j_{k_{\imath}}}$. Consider the product

$$
P=\prod_{j=1}^{k} \prod_{l=1}^{k_{\imath}} w_{i j_{l}^{2}}
$$

Initially, $P=1$. When an $\eta$-elimination occurs in the hypothesis $h_{i}$ for $C_{i}^{o p t}$, the weight $u_{i j}$ is reduced only if $h_{i}(x)=1$ and expert $E_{i}$ predicts wrongly on $x$. This can happen only at most $\eta$ times since we do a demotion on $w_{i j}$ only if $\alpha_{i j}<\eta$. Hence each $w_{i l}$ can only be divided by $\alpha$ at most $\eta$ times. On the other hand, each time a promotion occurs, $P$ is increased by $\alpha$ since $x$ is covered by at least one of the $C_{i}^{o p t}$, s, unless the expert whose competency region contains the instance $x$ predicts wrongly. The latter occurs at most $\eta$ times.

\footnotetext{
${ }^{7}$ A literal may be counted more than once in $L$ if it appears in more than one $C_{?}^{\circ p t}$.
} 
Therefore,

$$
\prod_{j=1}^{k} \prod_{l=1}^{k_{2}} w_{i j_{l}} \geq \alpha^{-\eta L} \alpha^{u-\eta}
$$

This implies

$$
\sum_{j=1}^{k} \sum_{l=1}^{k_{i}} \log _{\alpha} w_{i j_{l}} \geq u-\eta L-\eta .
$$

Note that $L=\sum_{i=1}^{k} k_{i}$. Thus, there exists a pair $i$ and $j$ such that

$$
\log _{\alpha} w_{i j} \geq \frac{u-\eta L-\eta}{L} .
$$

Theorem 8. Suppose a pool of $k$ experts having joint incompetence $\eta w . r . t$. the competency class of disjunctions of literals over a relevant subspace of the boolean. space $\{0,1\}^{n}$. Then we can identify the optimum covering in $O(n k)$ time per trial and making at most

$$
\frac{a}{a-1}+(1+\alpha k)\left(L\left(1+\eta+\log _{\alpha}(k n)\right)+\eta\right)
$$

mistakes. Here, $L$ is the sum, over all $C_{2}^{\text {opt }} s$, of the number of literals an the disjunction describing $C_{i}^{\text {opt }}$ and $\alpha$ is an arbitrary constant greater than 1 .

Proof: By Lemma 6 and Lemma 7 , there exists a pair $i$ and $j$ such that

$$
1+\log _{\alpha} k n \geq \log _{\alpha} w_{i j} \geq \frac{u-\eta L-\eta}{L} .
$$

Hence,

$$
u \leq L\left(1+\eta+\log _{\alpha} k n\right)+\eta
$$

Together with Lrmma 5, we get

$$
u+v \leq \frac{a}{\alpha-1}+(1+\alpha k)\left(L\left(1+\eta+\log _{\alpha} k n\right)+\eta\right) .
$$

Applying Theorem 8 to the example in Section 1.1 with $\alpha$ set to 2, we can make at most $22+15\left(m_{0}+m_{1}\right)+10 \log n$ mistakes instead of $2^{n}-\left|m_{0}-m_{1}\right|$ mistakes. However, it turns out that if we are interested in making predictions without regard to what the optimum covering is, then we can improve the mistake bound in Theorem 8 substantially.

The idea here is to treat the prediction of each expert $E_{i}$ on an instance $x$ as another variable $E_{i}(x)$. Suppose $D_{i}$ is the disjunctions describing the competency region of $E_{i}$ w.r.t. the optimum covering $C^{\text {opt }}$. Assuming that we predict 1 when at least one expert whose competency region contains $x$ predicts 1 , and 
0 otherwise. Then the hypothesis $h$ (of the target concept $f$ ) obtained by using $C^{o p t}$ for our prediction strategy on an instance $x$ corresponds to a disjunction

$$
\sum_{i=1}^{k} E_{i}(X) \cdot D_{i} .
$$

In other words, $h$ is a 2-DNF formula where each term is a conjunction of a literal and the variable $E_{i}(x)$ for some expert $E_{i}$.

WinNow2 can be used to agnostically learn disjunctions of $k$ literals with a mistake bound of $O(k \log N+\mathcal{M})$ [Lit88, Lit91, AW95] where $N$ is the number of variables and $\mathcal{M}$ is the number of mistakes made in the entire sequence of trials using the best disjunction. Thus, we can apply Winnow2 to learn $h$ agnostically and obtained a learning algorithm that has a smaller mistake bound.

Theorem 9. Suppose a pool of $k$ experts having joint incompetence $\eta$ w.r.t. the competency class of disjunctions of literals over a relevant subspace of the boolean space $\{0,1\}^{n}$. Then we can learn the competency class of disjunctions in $O(n k)$ time per trial and mistake bound $O(L \log (n k)+\eta)$.

\section{Hardness Result for Learning Competency Class of Conjunctions}

In this section, we reduce the problem of learning decision trees in the on-line mistake bound model to the problem of learning the competency class of conjunctions. In doing so, we show that if the competency class of linear threshold functions in the boolean domain, which contains the class of conjunctions, could be learned then we can learn decision trees as a concept class in the on-line mistake bound model. The latter problem, though not known to be impossible, is a difficult open problem.

Suppose we have a learner that learns the competency class of conjunctions. We illustrate below how we can make use of this learner to learn decision trees. The concept, and its complement, represented by a decision tree over the boolean domain can be expressed ${ }^{8}$ as DNF formulas where the number of terms is bounded by the number of leaves in the decision tree. Without loss of generality, let us assume we know how many terms there are in these two formulas. We associate each term in the formulas with one expert. Those experts that are associated with the terms in the target always predict positive while the rest always predict negative. The competency region of each expert is the term that it is associated with. Clearly, the terms cover the instance space and hence form a covering. It is also easy to see that the classification of an instance by the target decision tree is the same as the classification using this covering and the corresponding experts. Thus, we have the following theorem.

8 The complement of the target concept, $\bar{f}$, can be easily expressed as a DNF formula $g$ such that $g(x)=i$ if and only if $\overline{(} f)(x)=\bar{i}$. 
Theorem 10. If we could learn the competency class of conjunctions over $\{0,1\}^{n}$ then we can learn the class of decision trees in the mistake bound model.

\section{Learning the Competency Structure of Decision Lists}

In this section we present an algorithm that learns decision list competency structures over boolean instance spaces. More specifically, the optimum covering that we wish to learn is an ordered sequence $L$ of the form $\left\langle\left(a_{1}, E_{i_{1}}\right), \ldots\right.$ $\cdot \cdot\left(\alpha_{s-1}, E_{i_{s-1}}\right),\left(\alpha_{s}=\right.$ true,$\left.\left.E_{i_{s}}\right)\right\rangle$ where the $\alpha_{i}$ 's are literals and the $E_{i_{3}}$ 's are experts. Given an input $x \in\{0,1\}^{n}$ and the optimum covering $L$, the expert that we should use for predicting the classification of $x$ is $E_{i}$, such that $j$ is the smallest index satisfying $\alpha_{j}(x)=1$. For example, consider an instance $x=\langle 10010\rangle$ where $x_{0}$ and $x_{3}$ are set to 1 , and the other variables are set to 0 . If we were to use the covering $\left\langle\left(x_{1}, E_{4}\right),\left(\overline{x_{3}}, E_{1}\right),\left(\overline{x_{4}}, E_{3}\right),\left(x_{0}, E_{2}\right)\right\rangle$ as our strategy for making prediction, then we should use the advice of expert $E_{3}$.

We begin by assuming the incompetence of $\mathcal{E}$ is $\eta$. The idea here is to maintain a decision list $L^{\prime}$ which consists of $s$ consecutive sublists $L_{1}^{\prime}, \cdots, L_{s+1}^{\prime}$ where $s$ is the length of the optimum covering (decision list). Each sublist $L_{i}^{\prime}$ is a (possibly empty) ordered list $\left\langle\left(c_{l_{l}}, \mathcal{E}_{l_{1}}\right), \cdots,\left(c_{l_{2+1}-1}, \mathcal{E}_{l_{1+1}-1}\right)\right\rangle$ where the $\mathcal{E}_{l_{3}}$ 's is a duplicate copy of $\mathcal{E}$ instead of a single expert and the $c_{l}$, 's are the boolean literals. Furthermore, each boolean literal appears exactly once in $L^{\prime}$. Initially, all the sublists are empty except for $L_{1}^{\prime}$ which is set to

$$
\left\langle\left(x_{1}, \mathcal{E}\right),\left(\overline{x_{1}}, \mathcal{E}\right), \cdots,\left(x_{2}, \mathcal{E}\right),\left(\overline{x_{i}}, \mathcal{E}\right), \cdots,\left(x_{n}, \mathcal{E}\right),\left(\overline{x_{n}}, \mathcal{E}\right),(\text { truc }, \mathcal{E})\right\rangle .
$$

For each pair $\left(c_{j}, \mathcal{E}_{j}\right)$, we assigned an initial weight of one to each expert in $\varepsilon_{y}$. Given an instance $x$, we predict by running the weighted majority algorithm on $\mathcal{E}_{j}$ such that $j$ is the smallest index satisfying $c_{j}(x)=1$, and we say the rule $\left(c_{j}, \mathcal{E}_{j}\right)$ is fired. If a rule $\left(c_{j}, \mathcal{E}_{j}\right)$ in sublist $L_{l}$ fires and causes a mistake, we simply reduce the weights of those experts in $\mathcal{E}_{j}$ that predict wrongly from $\mathcal{E}_{j}$ by half. If we predict wrongly using the rule $\left(c_{j}, \mathcal{E}_{j}\right)$ more than $a(\log k+\eta)$ time while $\left(c_{j}, \mathcal{E}_{j}\right)$ remains in the same sublist $L_{l}^{\prime}$ then we move it to $L_{l+1}^{\prime}$ and reset the wcights of all the experts in $\mathcal{E}_{j}$ to 1 . Here $a$ is the constant in the mistake bound of the weighter majority algorithm (see Theorem 1a).

Let $\alpha_{i}$ denotes the literal in the $i$ th rule of our target optimum covering. It is clear that the rule $\left(\alpha_{1}, \mathcal{E}_{\bullet}\right)$ makes at most $a(\log k+\eta)$ mistakes while in sublist $L_{1}^{\prime}$ and thus, does not move beyond $L_{1}^{\prime}$. Similarly, the rule $\left(\alpha_{2}, \mathcal{E}_{\mathbf{0}}\right)$ can never move beyond $L_{2}^{\prime}$. A simple induction allows us to argue that the rule associated with $\alpha_{j}$ cannot move beyond $L_{j}^{\prime}$. Moreover, since each rule can make at most $a(\log k+\eta)$ mistakes while remaining in the same sublist, we can conclude that the total number of mistakes made is at most ans $(\log k+\eta)$.

Now, for unknown $\eta$, we can employ a standard doubling trick to guest the value of $\eta$ and run the above algorithm. Initially, our guess $\eta^{\prime}$ of $\eta$ is 1 and if the number of mistakes is more than ans $(\log k+\eta)$, we double the value of $\eta^{\prime}$. It is easy to show that the resulting mistake bound is $O(n s(\log k \log \eta+\eta))$. 
However, this requires us to know the length $s$ of the optimum covering (so that we known our guess is wrong when we make more than $O(n s(\log k \log \eta+\eta))$ mistakes). We can overcome this difficulty by running $\log (2 n+1)$ copies of the learning algorithm, $A_{1}, \cdots, A_{\log (2 n+1)}$ in turns where each $A_{i}$ assumes $s$ to be $2^{i}$. A simple argument shows that the mistake bound increases by at most a factor of $O(\log n)$. Thus, we have the following theorem.

Theorem 11. Given a pool of $k$ experts $\mathcal{E}$ with competency $\eta$ (w.r.t. the competency structure of decision lists), we can learn the competency structure of decision lists over $n$ boolean variables with mistake bound $O(n s \log n(\log k \log \eta+\eta))$. Here, $s$ is the length of the optimum covering and $\eta$ is the incompetence of $\mathcal{E}$ w.r.t. the competency structure of decision lists. The time complexity per trial of our algorithm is $O(n+k)$.

\section{Open Problems}

The competency model is well motivated, there are a number of fundamental open problems yet to be explored:

Problem 12. What are the competency classes or structures that can be learned or cannot be learned, beside those studied in this paper?

Problem 13. Perhaps the model is too general. Are there other well motivated variants that warrant investigation? If a competency class or structure cannot be learned, then does augmenting the model with a membership query oracle help? Is there a PAC-like version of the competency model where the instances are drawn according to a fixed distribution?

Problem 14. In this paper, we only consider boolean predictions. How about real predictions with other loss function like square loss or entropic loss?

Problem 15. In the competency class model, an instance $x$ may fall in the competency regions of more than one expert. How will the results change if we were to predict according to the majority vote of the experts whose competency regions contain $x$ ?

\section{Acknowledgements}

The authors like to thank the anonymous reviewers for their very valuable suggestions. Stephen Kwek is currently supported by NSF NYI Grant CCR-9357707 (of S. Goldman) with matching funds provided by Xerox PARC and WUTA.

\section{References}

[AW95] P. Auer and M. K. Warmuth. Tracking the best disjunction. In Proc. of the 36th Symposium on the Foundations of Comp. Sci., pages 312-321. IEEE Computer Society Press, Los Alamitos, CA, 1995. 
[CBFHW95] N. Cesa-Bianchi, Y. Freund, D. P. Helmbold, and M. K. Warmuth. Online prediction and conversion strategies. Machine Learning, 1995. To appear, an extended abstract appeared in Eurocolt ' 93.

[Lit88] N. Littlestone. Learning when irrelevant attributes abound: A new linearthreshold algorithm. Machine Learning, 2:285-318, 1988.

[Lit91] N. Littlestone. Redundant noisy attributes, attribute errors, and linear threshold learning using Winnow. In Proc. 4th Annu. Workshop on Comput. Learning Theory, pages 147-156, San Mateo, CA, 1991. Morgan Kaufmann.

[LW94] N. Littlestone and M. K. Warmuth. The weighted majority algorithm. Information and Computation, 108(2):212-261, 1994. 